\title{
ATENÇÃO PSICOSSOCIAL E SATISFAÇÃO NO TRABALHO: PROCESSOS DIALÉTICOS NA SAÚDE MENTAL ${ }^{1}$
}

\author{
PSYCHOSOCIAL ATTENTION AND SATISFACTION AT WORK: DIALECTICAL
}

PROCESSES IN MENTAL HEALT

\author{
Mara Cristina Ribeiro ${ }^{2}$ \\ Alice Correia Barros ${ }^{3}$ \\ Marinho da Silva Correia ${ }^{4}$ \\ Rebeca de Oliveira Lessa ${ }^{5}$ \\ Lucas Nascimento Tavares ${ }^{6}$ \\ Jéssica Bazilio Chaves ${ }^{7}$
}

Recebido em: 12 fev. 2017

Aceito em: 10 maio 2018

RESUMO: Introdução: $O$ modelo de atenção psicossocial tem exigido profissionais comprometidos com um novo paradigma de cuidado que traz novos e diferentes desafios, podendo gerar nos trabalhadores satisfação, insatisfação, bem como outros sentimentos que envolvem a efetivação das novas propostas. A satisfação ou a insatisfação com o trabalho incorre em consequências que podem ser fonte de alegria e bem-estar ou acarretar prejuízos à saúde do trabalhador e à qualidade do serviço prestado. Objetivo: A pesquisa teve como objetivo conhecer e analisar a satisfação e outros sentimentos gerados no exercício do trabalho nos Centros de Atenção Psicossocial. Método: Trata-se de pesquisa qualitativa desenvolvida com 19 trabalhadores de nível superior representantes de 04 serviços de uma capital do Nordeste. Os dados foram coletados por meio de entrevistas semiestruturadas e analisados com base na Análise Categorial. Resultados: A identificação com a área, a motivação em aprofundar seus conhecimentos e os bons resultados alcançados com as novas propostas de cuidado aparecem como determinantes para que

\footnotetext{
${ }^{1}$ A presente pesquisa foi desenvolvida pelo Programa de Educação pelo Trabalho (PET) - Saúde Mental da Universidade Estadual de Ciências da Saúde de Alagoas, aprovada pelo CEP sob o número de protocolo 1979/12. Foi apresentada no 11ํㅡㄹ Congresso Brasileiro de Saúde Coletiva, Goiânia, 2015.

2 Terapeuta Ocupacional, Doutora em Ciências, Professora Titular da Universidade Estadual de Ciências da Saúde de Alagoas. Maceió, Alagoas, Brasil. Endereço para correspondência: Mara Cristina Ribeiro. Centro de Ciências Integradoras - NUCISP/UNCISAL R. Dr. Jorge de Lima, 113 - Trapiche da Barra, Maceió - AL, 57010-300. Telefone: (82) 33251540. E-mail: maracrisribeiro@gmail.com.

${ }^{3}$ Enfermeira, Especialista em Saúde da Família, Residente em Psiquiatria e Saúde Mental. Universidade Estadual de Ciências da Saúde de Alagoas. Maceió, Alagoas, Brasil. E-mail: licinhabarros@hotmail.com.

${ }^{4}$ Enfermeiro. Universidade Estadual de Ciências da Saúde de Alagoas. Maceió, Alagoas, Brasil. E-mail: marinhoscorreia@hotmail.com.

${ }^{5}$ Enfermeira. Universidade Estadual de Ciências da Saúde de Alagoas. Maceió, Alagoas, Brasil. E-mail: bekalessa@hotmail.com.

${ }^{6}$ Acadêmico de medicina. Universidade Estadual de Ciências da Saúde de Alagoas. Maceió, Alagoas, Brasil.E-mail: luk_tav@hotmail.com.

7 Terapeuta Ocupacional, Mestre em Psicologia pela Universidade Federal de Alagoas. Maceió, Alagoas, Brasil.E-mail: jessicabazilioc@gmail.com.

Contribuições na elaboração do artigo

Barros AC, Correia MS, Lessa RO, Tavares NL e Chaves JB contribuíram com a produção dos dados, análise e interpretação dos resultados e elaboração do artigo até a sua versão final.

Ribeiro MC contribuiu na concepção do trabalho, orientação, análise e interpretação dos resultados e revisão crítica do artigo e aprovação final da versão a ser publicada.
} 
o trabalhador se sinta satisfeito. No entanto, constatou-se que os profissionais se deparam cotidianamente com problemas que foram associados à geração de insatisfações e outros sentimentos como angústia, incapacidade, tristeza e frustração. Conclusão: As questões levantadas sugerem necessidade de maiores investimentos nesses serviços, capacitações dos profissionais, ampliação e diversificação da rede de atenção psicossocial para garantir satisfação no exercício do trabalho e na qualidade da assistência prestada.

Palavras-chave: Saúde mental. Serviços de Saúde Mental. Pessoal de Saúde. Satisfação no Emprego. Pesquisa Qualitativa.

ABSTRACT: Introduction: The psychosocial care model has required professionals committed to a new care paradigm that brings new and different challenges, which can generate satisfaction in workers, dissatisfaction, and other feelings that involve the execution of the new proposals. The satisfaction or dissatisfaction with the work incurs consequences that can be a source of joy and well-being or cause damage to workers' health and quality of service. Objective: The objective was to identify and analyze the satisfaction and other feelings generated in the performance of work in Psychosocial Care Centers. Method: This is a qualitative research conducted with 19 top-level workers representatives from 04 of the capital of Northeast services. Data were collected through semi-structured interviews and analyzed based on Categorical Analysis. Results: Identification with the area, the motivation to deepen their knowledge and the good results achieved with the new care proposals appear as decisive for the worker to feel satisfied. However, it was found that professionals are faced daily with problems that were associated with the generation of dissatisfactions and other feelings such as anxiety, failure, sadness and frustration. Conclusion: The issues raised suggest the need for greater investment in these services, training of professionals, expansion and diversification of psychosocial care network to ensure satisfaction in work performance and quality of care.

Keywords: Mental Health. Mental Health Services. Health Personnel. Job Satisfaction. Qualitative Research.

\section{INTRODUÇÃO}

A política nacional de saúde mental tem investido na expansão contínua dos Centros de Atenção Psicossocial (CAPS), entendendo-os como serviços estratégicos da Rede de Atenção Psicossocial (RAPS) para a superação do modelo asilar (ASSIS et al.,2014). Seus objetivos não se limitam ao atendimento médico e psicológico, respondem à perspectiva da construção de um território de atenção, tecido na rede de cuidados à saúde mental, garantindo o acesso e a equidade, produzindo autonomia e direito aos seus usuários por meio da assistência realizada por equipe multidisciplinar.

Desta forma, têm por base um trabalho comunitário, humanizador e reintegrador do homem no contexto social, trazendo nova significação individual e social para as pessoas que os frequentam (SCHNEIDER et al., 2009).

Segundo Zerbetto et al (2011) a tendência atual é a construção e o fortalecimento de um novo paradigma de cuidado, o da atenção psicossocial, que pressupõe ações não só nas esferas da concepção do processo saúde-doença mental mas também nos meios teórico-técnicos, nas organização das relações intrainstitucionais, nas relações da 
instituição e seus agentes com a clientela e com a população em geral.

Para tanto, os CAPS dispõem de equipe multidisciplinar em ações interdisciplinares e intersetoriais; oferecendo atendimentos individuais e grupais, oficinas terapêuticas, oficinas de geração de renda, atividades lúdicas e desportivas, tratamento medicamentoso, atendimento familiar, articulação com a rede e com o território, entre outras estratégias, visando garantir a construção de um lugar social aos seus usuários e estimular seu protagonismo frente à vida (RIBEIRO, 2013).

A Portaria no 854 de 2012 amplia as ações desenvolvidas por este serviço, incluindo a promoção de contratualidade no território, ações de reabilitação psicossocial e redução de danos, matriciamento e articulação de redes intra e intersetoriais (ASSIS et al., 2014).

Embora o modelo de atenção exija profissionais comprometidos com o paradigma da atenção psicossocial, estes, em suas práticas cotidianas, encontram grande dificuldade, uma vez que não possuem em sua formação os subsídios necessários para a compreensão desse novo modelo (RIBEIRO, 2015).

Estudos têm evidenciado grandes lacunas entre a formação dos trabalhadores e as exigências práticas nos CAPS, demonstrando a dificuldade do trabalhador em conciliar o seu aprendizado teórico com os desafios do cotidiano que o novo cuidado em saúde mental incita (RIBEIRO, 2015; FILIZOLA, MILIONI, PAVARINI, 2008). Estas dificuldades, entre outras, podem gerar nos trabalhadores falta de motivação no desenvolvimento do processo de trabalho e na qualidade de vida dos mesmos.

Para Athayde e Hennington (2012), o campo da saúde ocupacional vem oferecendo contribuições importantes para o entendimento das repercussões do trabalho sobre a saúde e o bem-estar de profissionais. Apesar da relevância do tema, a produção científica sobre a satisfação dos trabalhadores que atuam nesses serviços ainda é escassa.

Estar ou não satisfeito com o trabalho incorre em consequências diversas, sejam elas no plano pessoal ou profissional, afetando diretamente o comportamento, a saúde e o bem-estar do trabalhador. A satisfação no trabalho pode ser, por conseguinte, fonte de saúde, bem como a insatisfação pode gerar prejuízos à saúde física, mental e social, acarretando problemas à organização, ao ambiente de trabalho (MARQUEZE, MORENO, 2005) e, consequentemente, um prejuízo no que diz respeito à qualidade da assistência prestada ao usuário.

Há que se considerar as transformações ocorridas no mundo do trabalho e os movimentos de reforma do setor saúde, os quais se configuram como processos promotores de mudanças na gestão e na organização dos processos de trabalho em saúde e, nesse caso específico, em saúde mental. Assim, os trabalhadores estão expostos a diversas situações, as quais podem ocasionar maior ou menor satisfação.

Portanto, conhecer se há ou não satisfação no exercício das novas práticas e se a isso somam-se outros sentimentos, gera subsídios para a criação de estratégias que possam melhorar a qualidade de trabalho $e$ de vida dos trabalhadores e, 
consequentemente, contribuir com a qualidade dos serviços prestados.

Desta forma, desenvolveu-se esta pesquisa, cujo objetivo foi conhecer e analisar a satisfação e outros sentimentos gerados no exercício do trabalho desenvolvido nos CAPS, tendo em vista que as novas estratégias de trabalho utilizadas nesses serviços exigem de seus trabalhadores diferentes protagonismos.

\section{MATERIAL E MÉTODOS}

A pesquisa qualitativa é interpretativa, baseada em experiências, situacional e humanística, desta forma, as atividades e contextos selecionados para se investigar são escolhidos por oferecem possibilidades de compreensão de como alguma realidade funciona, considerando as experiências como revelações perspicazes (STAKE, 2011). Portanto, para alcançar os objetivos propostos desse estudo optou-se pela abordagem qualitativa.

A investigação foi desenvolvida em quatro Centros de Atenção Psicossocial de uma capital da região nordeste do Brasil. Foram sujeitos do estudo 19 trabalhadores desses serviços, com formação superior, das mais diversas categorias profissionais. Estes foram convidados a participar do estudo na reunião técnica dos serviços, após a explicação sobre a pesquisa e seus objetivos. Todos que se mostraram disponíveis foram contatados em momento posterior e, respeitando o critério de intencionalidade - que permitiu a variação das formações profissionais dos entrevistados - foram marcadas entrevistas individuais.

Para a coleta dos dados foi utilizada a técnica de entrevista semiestruturada com o uso de formulário contendo questões fechadas, que permitiram conhecer o perfil dos participantes e a criação de um gráfico sobre o nível de satisfação dos trabalhadores, e questões abertas, que permitiram avaliar de forma mais aprofundada a visão dos entrevistados sobre suas satisfações, insatisfações e outros sentimentos gerados no exercício do trabalho. O período destinado para a esta etapa foi entre maio a agosto de 2013.

O método utilizado para analisar as informações obtidas foi a Análise de Conteúdo, em sua modalidade Análise Categorial, percorrendo os caminhos de pré-análise, exploração do material e tratamento dos resultados, e por fim sua interpretação e possíveis inferências (BARDIN, 2011).

Todos os aspectos éticos que envolvem investigação com seres humanos foram respeitados durante o trâmite da pesquisa, atendendo à Resolução no 466/2012 do Conselho Nacional de Saúde. O estudo foi aprovado pelo Comitê de Ética e Pesquisa sob o número de protocolo 1979/12. Todos os participantes, após esclarecimentos acerca dos objetivos da pesquisa e da garantia de preservação de suas identidades, assinaram o Termo de Consentimento Livre e Esclarecido. Para a apresentação dos resultados foram atribuídas siglas aos nomes dos sujeitos entrevistados a fim de preservar sua identidade. 


\section{RESULTADOS}

Foram entrevistados 19 profissionais de nível superior de diferentes formações, a Tabela 1 apresenta a quantidade correspondente por formação dos entrevistados. Destes entrevistados, à época do estudo, 13 possuíam apenas ensino superior completo, 05 completaram pós-graduação lato sensu e 01 possuía doutorado. Seis profissionais tinham complementação específica em saúde mental como capacitação (2), especialização (3) e residência e doutorado em psiquiatria (1).

Tabela 1. Formação dos entrevistados

\begin{tabular}{lc}
\hline FORMAÇÃO & QUANTIDADE \\
\hline Enfermagem & 04 \\
Psicologia & 03 \\
Terapia Ocupacional & 03 \\
Medicina & 02 \\
Farmácia & 02 \\
Fisioterapia & 01 \\
Fonoaudiologia & 01 \\
Nutrição & 01 \\
Educação Física & 01 \\
Teatro & 01 \\
\hline
\end{tabular}

Fonte: Dados da Pesquisa

A média de idade foi de 38 anos e meio, com mínimo de 25 e máximo de $65 ; 16$ dos entrevistados eram do sexo feminino.

Com relação à caracterização dos trabalhadores segundo o tempo de serviço, os participantes do estudo possuíam tempo médio de trabalho em saúde mental de 6 anos e 8 meses, sendo o tempo de trabalho específico nos CAPS de 5 anos e 4 meses.

Para a análise das falas foram estabelecidas categorias para a melhor compreensão dos temas encontrados. Desta forma, após as leituras de assimilação, utilizando critérios de relevância e de repetição e tendo o objetivo da pesquisa como pano de fundo, foram estabelecidas duas categorias:

1. A (in) satisfação profissional em relação ao trabalho exercido no CAPS e seus motivos;

2. Outros sentimentos despertados durante o trabalho no CAPS e seus motivos.

Categoria 1: Satisfação ou insatisfação profissional em relação ao trabalho exercido no CAPS e seus motivos

Esta primeira categoria apresenta a percepção de satisfação ou insatisfação dos 
profissionais no exercício do trabalho nos CAPS, tendo como preocupação não somente a apresentação do nível de satisfação dos respondentes, mas também a compreensão das motivações acerca desses sentimentos.

Desta forma, ao responderem as questões relativas à satisfação com o trabalho que exercem no CAPS, os entrevistados, inicialmente, tiveram a opção de responder, por meio de uma pergunta fechada, se sentiam-se muito satisfeitos, satisfeitos, um pouco satisfeitos ou insatisfeitos. Uma parte significativa dos profissionais respondeu que se sente satisfeito com o trabalho, o que pode ser verificado na Figura 1.

Figura 1: Representação do nível de satisfação dos profissionais em relação ao trabalho exercido no CAPS.

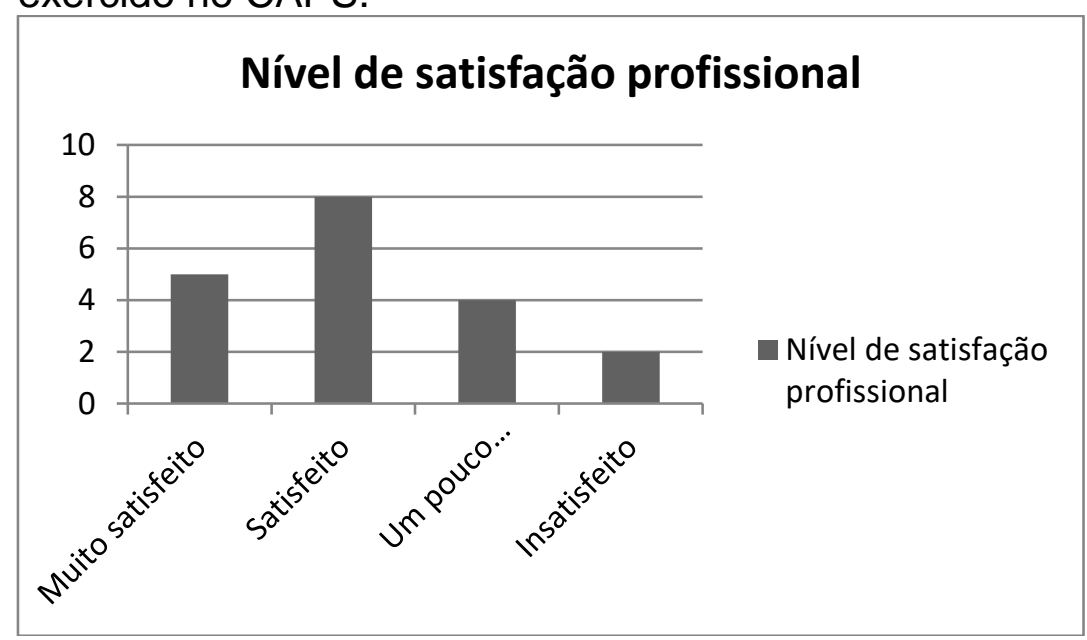

Fonte: Dados da pesquisa

Após essa questão fechada, o estudo procurou conhecer, por meio de uma questão aberta, as razões que sustentavam suas respostas.

A identificação com a área de trabalho e os resultados positivos alcançados pelo serviço foram apontados como fatores que geram satisfação.

[...] acho que é muito satisfatório você ver o paciente falando de como era e como está, o que o CAPS fez por ele. (P11)

Eu tenho me identificado e tenho ficado satisfeita, não muito satisfeita ainda, porque eu gostaria de ter mais condições de exercer este trabalho e até me formar melhor também estando aqui nesta área específica. (P8)

[...] é extremamente gratificante, é um trabalho que a gente tá trocando sempre informação, estamos chegando perto de uma população que, geralmente, não tem um acesso fora. (P4)

No entanto, é interessante apontar que, apesar de 13 dos respondentes afirmarem muita satisfação ou satisfação com o trabalho exercido no serviço, ao serem convidados a explicar os motivos da satisfação, muitos expressaram contradição, pois apresentaram considerações que demonstravam certo grau de insatisfação.

Desta forma, ao mesmo tempo em que encontramos nas falas daqueles que disseram estar muito satisfeitos ou satisfeitos ponderações como:

Acho que o CAPS tem grandes possibilidades de fazer um trabalho efetivo [...]. (P6) 
É um trabalho que a gente está trocando sempre informações, estamos chegando perto de uma população que geralmente não tem um acesso fora de um meio como esse [...]. (P4)

Porque eu gosto de trabalhar aqui, eu me identifiquei muito com o serviço, com os usuários. (P19)

Eu me capacitei sozinha, em estudar, pesquisar, ver como é o andamento de uma pessoa com transtorno mental, e hoje eu posso dizer que estou satisfeita. (P12)

Também foi possível encontrar, entre estes mesmos respondentes, falas como as abaixo:

É muito desmotivante vir pra cá todos os dias e você não ter perspectiva nem de melhora. (P19)

Tem vezes de eu querer sair pra ir pra outro serviço, só que com o passar do tempo eu aprendi a lidar com as situações [...]. (P12)

[...] porque eu não consigo fazer tudo o que eu queria fazer [...] porque somos muito dependentes de outras coisas. (P16)

Com relação a pouca satisfação ou insatisfação, os entrevistados justificam suas respostas relacionando-as a faltas gerais: falta de estrutura, de material, de investimento, de reconhecimento da importância do serviço perante a gestão municipal, de articulação da rede, entre outras.

Não tem espaço adequado, não tem material adequado... Não propicia um atendimento adequado. (P1)

Insatisfeito! Porque a gente tem muito pouca resolutividade nas ações, há muito pouco investimento da saúde mental municipal, da saúde municipal de uma forma geral. A rede é pouco articulada, o que existe é pouco articulado e muitos pontos que deveriam existir da rede, não existem. (P9)

[...] falta material, falta estrutura física, falta capacitação, então você acaba se desmotivando cada vez mais. (P18)

As falas, ainda, tornam evidente o desejo de mudanças no sentido de possibilitar a realização dos objetivos que os CAPS se propõem e que os seus trabalhadores esperam executar.

Porque mesmo a gente querendo fazer, a gente não consegue fazer $100 \%$ daquilo que a gente quer. Aí deixa a sensação de insatisfação, mesmo... porque a gente não consegue demonstrar o que a gente pode fazer. (P13)

A gente não consegue fazer um trabalho de matriciamento, a gente não consegue fazer um trabalho de inserção territorial, e é muito difícil fazer o trabalho que o CAPS deveria fazer [...] então a gente tem muita dificuldade com relação a isso, por isso a insatisfação. (P9)

\section{Categoria 2: Outros sentimentos despertados durante o trabalho no CAPS e seus}

\section{motivos}

Nessa categoria, são expostos outros sentimentos gerados a partir das práticas exercidas nos CAPS, ou seja, para além dos sentimentos de satisfação ou insatisfação, o estudo procurou conhecer que outras emoções o exercício do trabalho no CAPS desperta.

Nas falas dos profissionais entrevistados, pode-se perceber a presença de sentimentos positivos. 
É extremamente gratificante, é um trabalho que a gente está trocando sempre informação. (P4)

[...] Eu me sinto realizada como pessoa, como profissional, resumindo, como tudo! (P5) Eu acho assim, que trabalhar com saúde mental é tudo de bom, se você focar assim para sua própria vida, então você diminui o seu sofrimento com o sofrimento das outras pessoas, entendeu? Eu acho que contribui muito para a sua vida pessoal. (P12)

No entanto, foram também citados aspectos negativos como sentimentos de angústia, incapacidade, tristeza, frustração.

É um sinal de que eu não sou, como se fosse incapacidade, é um sentimento de incapaz, você tenta fazer alguma coisa, porque o trabalho não só depende de mim. (P7)

[...] tristeza com muitas, tem muita história triste, muito abandono, descaso é, muita... enfim, é, é a tristeza e a ansiedade é pela falta de condições de trabalho mesmo. (P9)

Frustração. Acho que o maior sentimento é o de frustração. (P18)

É angustiante, imagine a frustração do profissional em você querer ver um usuário que você sabe que você tem condições dele ter uma melhora substancial e você fica de mãos atadas, porque só com o trabalho humano as vezes você não consegue atingir os objetivos que você quer, porque haja criatividade de só aqui com o corpo estar trabalhando com eles diariamente. (P19)

Estes e outros relatos indicam que a falta de estrutura, somada ao pouco investimento na qualificação dos trabalhadores e as deficiências da rede de assistência à saúde mental geram consequências graves no exercício do trabalho, pois acarretam sentimentos bastante nocivos ao trabalhador.

Ao serem questionados a respeito dos motivos que justificam os sentimentos expostos acima, evidenciou-se que os entrevistados, apesar de sentirem-se satisfeitos e gostarem de suas práticas, sentem frustração por quererem realizar o trabalho e ao mesmo tempo terem que lidar com a falta de condições no serviço e de investimento do poder público na área da saúde mental.

Eu gosto de trabalhar aqui, me identifiquei muito com o serviço, com os usuários, mas o que está faltando pra gente há muito tempo é estrutura, a gente não tem nada pra trabalhar mesmo [...] e não ter ideia do que você vai trabalhar naquele dia porque não tem material básico que é caneta, papel, lápis, então você tem que se virar em mil pra poder ter uma ideia do que vai fazer. (19)

Primeiro a clientela... é uma clientela difícil... além do transtorno em si, tem a questão social, a questão do ambiente, tem a família... a questão do serviço em si... não tem espaço adequado, não tem material adequado... não propicia um atendimento adequado. (P14)

Porque você quer realizar e não consegue, tem esse entrave, principalmente do poder público, [...] que a saúde mental é uma área que não é valorizada na saúde. Sempre é excluída. (P18)

\section{DISCUSSÃO}

Sabemos que satisfação no trabalho é uma condição determinada por aspectos intrínsecos e extrínsecos ao ambiente (GUIMARÃES, JORGE, ASSIS, 2011) e, portanto, faz sentido a consideração de diferentes enfoques que resultam na geração desse sentimento ou de seu contrário. 
A identificação com a área, a motivação em aprofundar os conhecimentos e os bons resultados alcançados com as novas propostas de cuidado podem ser determinantes para que o trabalhador sinta-se estimulado e, portanto, satisfeito.

O trabalho em equipe e o contato com o usuário também foram elencados como razões de satisfação no exercício do trabalho, corroborando com estudos anteriores sobre a temática (RIBEIRO, 2015; REBOUÇAS, LEGAY, ABELHA, 2007).

No entanto, foi possível detectar que muitos, ao serem convidados a refletir sobre a sua satisfação com relação ao trabalho se contradizem exatamente porque diversas perspectivas se envolvem nessa avaliação. Assim, o que de imediato foi respondido como satisfação ou muita satisfação no trabalho, transforma-se por meio de um aprofundamento sobre as razões dessa satisfação em outros sentimentos, trazendo à tona aspectos ligados às desmotivações geradas pela própria exposição a situações de estresse diário, que são características comuns nos CAPS.

A saúde mental é um campo de conhecimento e de atuação técnica bastante complexo que vem exigindo de seus trabalhadores atuarem com novas tecnologias de cuidado, ações integradas da equipe, relações horizontais com usuários, familiares e aproximação real com a comunidade. Estas novas demandas do cuidado podem acarretar ao trabalhador compromissos mais efetivos com o sucesso do tratamento, por ser o trabalhador, nessas novas proposições, protagonista das transformações do cuidado e da qualidade dos serviços.

Portanto, é possível inferir que o alcance ou não dos objetivos aos quais o exercício do trabalho se propõe, se apresenta como determinante na avaliação de satisfação.

Importante pontuar que aqueles que se colocaram de imediato como insatisfeitos ou um pouco satisfeitos, relacionam essa avaliação diretamente aos resultados que não podem ser alcançados devidos às faltas materiais e estruturais percebidas no dia-a-dia dos serviços.

São estas mesmas faltas que geram, segundo nossos entrevistados, outros sentimentos além da insatisfação, tais como angústia, tristeza e incapacidade.

Estudos realizados em outras regiões do país também têm indicado a falta de estrutura física e pouco investimento como fatores que impulsionam a insatisfação dos trabalhadores da área de saúde mental (REBOUÇAS, LEGAY, ABELHA, 2007; De MARCO et al, 2008; GUIMARÃES, JORGE, ASSIS, 2011).

Outros estudos têm apontado que as gestões municipais, de uma maneira geral, não assumem de forma integral as propostas do Ministério da Saúde e este fato gera a falta de investimento nos serviços, indicando que o reflexo disso são serviços com estruturas físicas precárias, falta de material para as intervenções terapêuticas, bem como equipes pouco investidas no que se refere às capacitações e ações de qualificação do serviço (RIBEIRO, 2016; LUZIO, L'ABATTE, 2009).

Quando falamos no Sistema Único de Saúde (SUS), em especial os serviços de 
saúde mental, parece-nos muitas vezes que estamos muito distantes de alcançar as diretrizes fundamentadoras e os pilares que sustentam suas práticas. Percebe-se que a saúde mental na saúde publica vivencia momentos de fragilidade onde devemos levar em consideração qualquer determinante que venha abalar ainda mais os serviços ofertados por esse sistema.

A ênfase na atenção à satisfação do trabalhador é bastante importante para o alcance dos objetivos do CAPS, pois, o campo psicossocial pressupõe ação integrada da equipe de trabalho, baseada em princípios de solidariedade, acolhimento, cooperação em torno da produção de saúde e cuidado compatíveis com as necessidades dos usuários (SILVA, FONSECA, 2005).

Mesmo considerando os avanços na construção do modelo de atenção psicossocial, as equipes dos serviços de saúde mental têm sido marcadas pela emergência de contradições ligadas aos processos de implantação desses equipamentos, que repercutem nos processos de trabalho, nas relações estabelecidas entre os diferentes atores e, na satisfação, prazer e sofrimento dos trabalhadores (SAMPAIO et al 2011).

Os relatos que emergiram dessa pesquisa demonstram que os profissionais que atuam na área da saúde mental sentem dificuldade em prestar uma assistência de qualidade, pois apesar de sentirem-se realizados profissionalmente e gostarem do trabalho, são obrigados a lidar com uma inversão de investimentos (afetivos, materiais e estruturais) em seu cotidiano, que traz, consequentemente, desestímulo, angústia e tristeza.

Desta forma, as dificuldades profissionais no trabalho acabam por interferir na assistência prestada, pois fazem emergir sentimentos como a frustração, a sensação de fracasso e a impotência que impedem o profissional de exercer o seu papel de forma adequada, no sentido de atender às necessidades do usuário nos seus aspectos biopsicossociais (MARQUEZE, MORENO, 2005).

Nos discursos, evidencia-se a presença de diversos sentimentos advindos de um ambiente de trabalho com muita dificuldade estrutural, material e funcional, que caracteriza comportamento de insatisfação profissional e geram dificuldades em lidar com o dia-a-dia no trabalho. O profissional insatisfeito no seu ambiente de trabalho pode desenvolver problemas psicológicos, profissionais e emocionais, que poderão afetar a qualidade do atendimento e dos serviços prestados ao usuário.

Portanto, caminhar entre um cotidiano habitado de sentimentos contraditórios, repleto de satisfações e insatisfações, expectativas e frustrações, potências e impotências, pode exaurir forças e tornar árduo o exercício das práticas psicossociais.

\section{CONSIDERAÇÕES FINAIS}

Os CAPS são serviços que surgiram no contexto da reforma psiquiátrica, criados para romper com a estrutura alienante dos hospitais psiquiátricos, bem como evitar a 
segregação do usuário dos serviços de saúde mental e as formas opressoras de tratamentos que o mesmo era submetido. Estão fundamentados na humanização do serviço, nos processos de trabalho desenvolvidos e na atenção integrada à comunidade.

Aos trabalhadores que neles atuam é necessário que estejam engajados com novas tecnologias de cuidado para que possam colocar em prática a integralidade dos cuidados oferecidos, bem como consigam atuar com a subjetividade da saúde mental e as transformações que ocorrem no setor da saúde como um todo.

No entanto, o estudo permitiu constatar que embora muitos trabalhadores se percebam satisfeitos com o trabalho em saúde mental e com as novas práticas exigidas nesse contexto, este mesmo trabalho tem gerado insatisfações das mais diversas ordens.

Evidenciaram-se alguns pontos determinantes de insatisfação como a estrutura física e a falta de manutenção nos ambientes, falta de material de suporte para desenvolvimento das atividades profissionais e falta de capacitação em saúde mental para os trabalhadores que atuam nos CAPS.

O incremento, a ampliação e a diversificação da rede atual de atenção à saúde mental também devem ser priorizados, para tanto, o envolvimento dos gestores é decisivo. A saúde mental deve fazer parte de investimentos maiores nas gestões municipais, para que o trabalhador possa, de fato, realizar a interlocução dos usuários dos serviços com a coletividade, cumprindo, dessa forma, os objetivos que a política de saúde mental se propõe.

Acredita-se que a construção de práticas efetivas do cuidado dentro da perspectiva da atenção psicossocial precisa, necessariamente, atentar para a saúde mental de todos os atores envolvidos e, portanto, as necessidades, expectativas e sofrimentos despertados no trabalho de seus profissionais devem ser considerados, para que, a partir da apuração dessas tensões, possam ser introduzidos elementos nessas práticas que gerem processos de satisfação, alegria e outros sentimentos de bem-estar no trabalho.

\section{REFERÊNCIAS}

ASSIS, Jaqueline Tavares et al. Política de saúde mental no novo contexto do Sistema Único de Saúde: regiões e redes. Divulgação em Saúde para Debate, v. 52, p.88-113, 2014.

ATHAYDE, Vladimir; HENNINGTON, Élida Azevedo. A saúde mental dos profissionais de um centro de atenção psicossocial. Physis, v. 22, n. 3, p. 983-1001, 2012. Disponível em: <http://www.redalyc.org/articulo.oa?id=400838257008>

BARDIN, Laurence. Análise de conteúdo. São Paulo: Edições 70; 2011.

DE MARCO, Patrícia Furuta et al. O impacto do trabalho em saúde mental: transtornos psiquiátricos menores, qualidade de vida e satisfação profissional. Jornal Brasileiro de Psiquiatria, v. 57, n.3, p.178-183, 2008. Disponível em: 
http://www.scielo.br/pdf/jbpsiq/v57n3/04.pdf

FILIZOLA, Carmen Lúcia Alvez; MILIONI, Débora Brechesi; PAVARINI, Sofia Cristina lost. A vivência dos trabalhadores de um CAPS diante da nova organização do trabalho em equipe. Revista Eletrônica de Enfermagem, v. 10, n. 2, p. 491-503, 2008. Disponível em: http://www.fen.ufg.br/revista/v10/n2/v10n2a20.htm

GUIMARÃES, José Maria Ximene; JORGE, Maria Salete Bessa; ASSIS, Marluce Maria Araújo. (In)Satisfação com o trabalho em saúde mental: um estudo em centros de atenção psicossocial. Ciência \& Saúde Coletiva, v.16, n. 4, p. 2145-54, 2011. Disponível em: http://www.scielo.br/pdf/csc/v16n4/v16n4a14.pdf

LUZIO, Cristina Amélia; L'ABATTE, Solange. A atenção em Saúde Mental em municípios de pequeno e médio portes: ressonâncias da reforma psiquiátrica. Ciência \& Saúde Coletiva, v. 14, n. 1, p. 105-116, 2009. Disponível em:

http://www.scielo.br/scielo.php?script=sci_arttext\&pid=S1413-81232009000100016

MARQUEZE, Elaine Cristina; MORENO, Claudia Roberta de Castro. Satisfação no trabalho - uma breve revisão. Revista Brasileira de Saúde Ocupacional. v. 30, n. 112, p. 69-79. 2005. Disponível em: http://www.scielo.br/scielo.php?script=sci_arttext\&pid=S030376572005000200007

MERHY, Emerson Elias. Os CAPS e seus trabalhadores no olho do furacão antimanicomial. Alegria e alívio como dispositivos analisadores. In: AMARAL, Heloisa; MERHY, Emerson Elias. (organizadores). A Reforma psiquiátrica no cotidiano II. São Paulo: Aderaldo \& Rothschild; 2007. p.55-66.

REBOUÇAS, Denise; LEGAY, Letícia Fortes; ABELHA, Lúcia. Satisfação com o trabalho e impacto causado nos profissionais de serviço de saúde mental. Revista de Saúde Pública, v. 41, n. 2, p. 244-50, 2007. Disponível em:

http://www.scielo.br/pdf/rsp/v41n2/5992.pdf

RIBEIRO, Mara Cristina. Os Centros de Atenção Psicossocial como espaços promotores de vida: relatos da desinstitucionalização em Alagoas. Revista de Terapia Ocupacional da Universidade de São Paulo, v. 24, n. 3, p. 174-82, 2013.

DOI: http://dx.doi.org/10.11606/issn.2238-6149.v24i3p174-182

RIBEIRO, Mara Cristina. Trabalhadores dos Centros de Atenção Psicossocial de Alagoas: interstícios de uma nova prática. Interface - Comunicação Saúde Educação, Botucatu, v. 19, n. 52, p. 95-107, 2015. DOI: http://dx.doi.org/10.1590/1807-57622014.0151

SAMPAIO, José Jackson Coelho et al. O trabalho em serviços de saúde mental no contexto da reforma psiquiátrica: um desafio técnico, político e ético. Ciência \& Saúde Coletiva, v. 16, n. 12, p. 4685-94, 2011. Disponível em:

http://www.scielo.br/pdf/csc/v16n12/17.pdf

SCHNEIDER, Jacó Fernando et al.. Avaliação de um centro de atenção psicossocial brasileiro. Ciencia y Enfermeria. Concepción, v. 15, n. 3, p. 91-100, 2009. Disponível em: http://www.scielo.cl/pdf/cienf/v15n3/art_10.pdf

SILVA, Ana Luíza Aranha; FONSECA, Rosa Maria Godoy Serpa. Processo de trabalho em saúde mental e o campo psicossocial. Revista Latino-Americana de Enfermagem , 
v.13, n.3, p. 441-9, 2005. Disponível em:

http://www.scielo.br/scielo.php?script=sci_arttext\&pid=S0104-11692005000300020

STAKE, R. E. Pesquisa qualitativa: estudando como as coisas funcionam. Porto Alegre: Penso, 2011

ZERBETTO, Sonia Regina et al. O trabalho em um centro de atenção psicossocial: dificuldades e facilidades da equipe de enfermagem. Revista Eletrônica de Enfermagem, v. 13 n. 1, p. 99-109, 2011. Disponível em:

https://www.fen.ufg.br/fen_revista/v13/n1/v13n1a11.htm 